\title{
iGrasp Robotic Prosthetic Hand
}

Figure 1. CAD Model of the iGrasp Hand

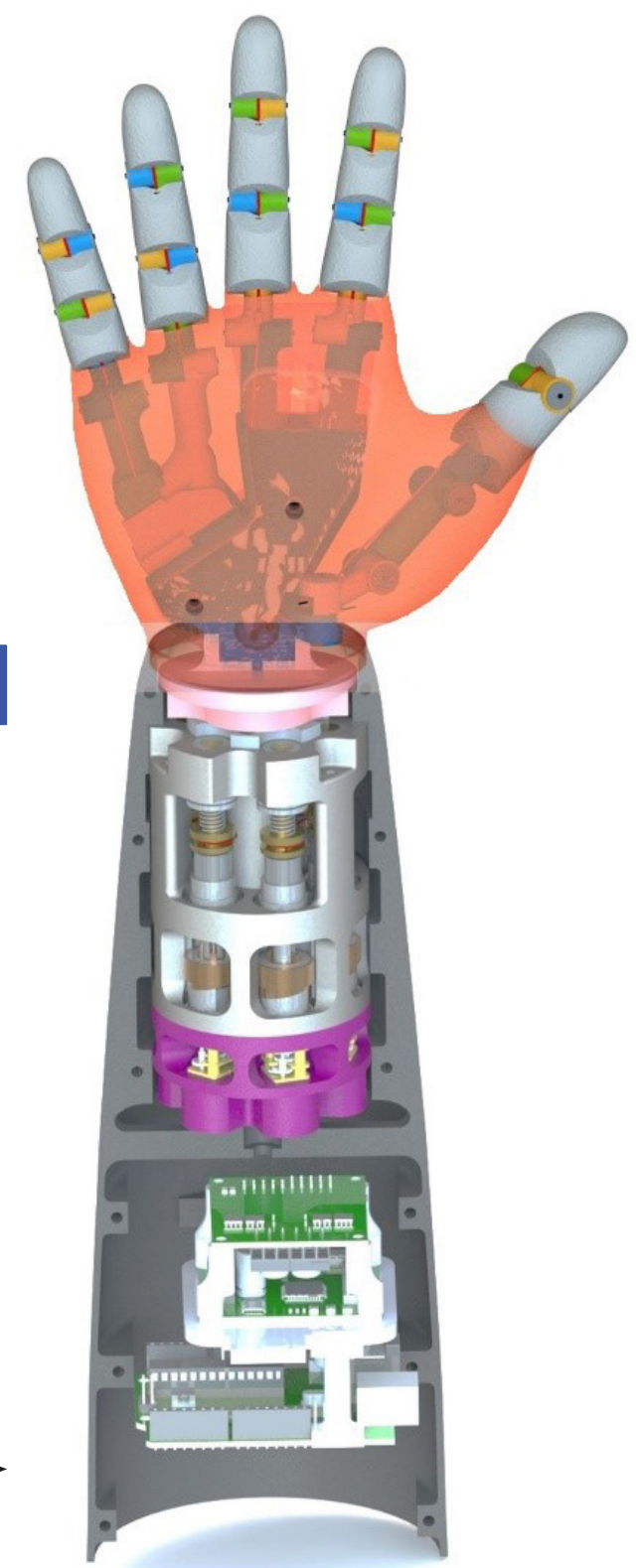

We are living in an era in which technology is shaping the world at an incredible speed. In this wake, the Bionics laboratory of the Department of Mechanical Engineering of the University of Moratuwa is doing inspiring research to improve the quality of life of differently-abled people. The latest product of this effort emerged as the iGrasp Hand, a robotic prosthetic hand that can mimic the grasping patterns of a human hand.

The focus of the iGrasp Hand is trans-radial amputees, the amputees who have undergone partial removal of the forearm due to trauma or other conditions. Such amputations restrict a person's mobility and activities of daily living, instilling a plethora of challenges that have forced the technology behind the transradial prostheses to shift from merely cosmetic devices to complex, biomimetic devices over the ages. Commercial prostheses such as Bebionic [1], i-Limb [2], and Michelangelo prosthetic hand [3] are a few biomimetic devices that have captured the essence of the human hand to a certain degree. 


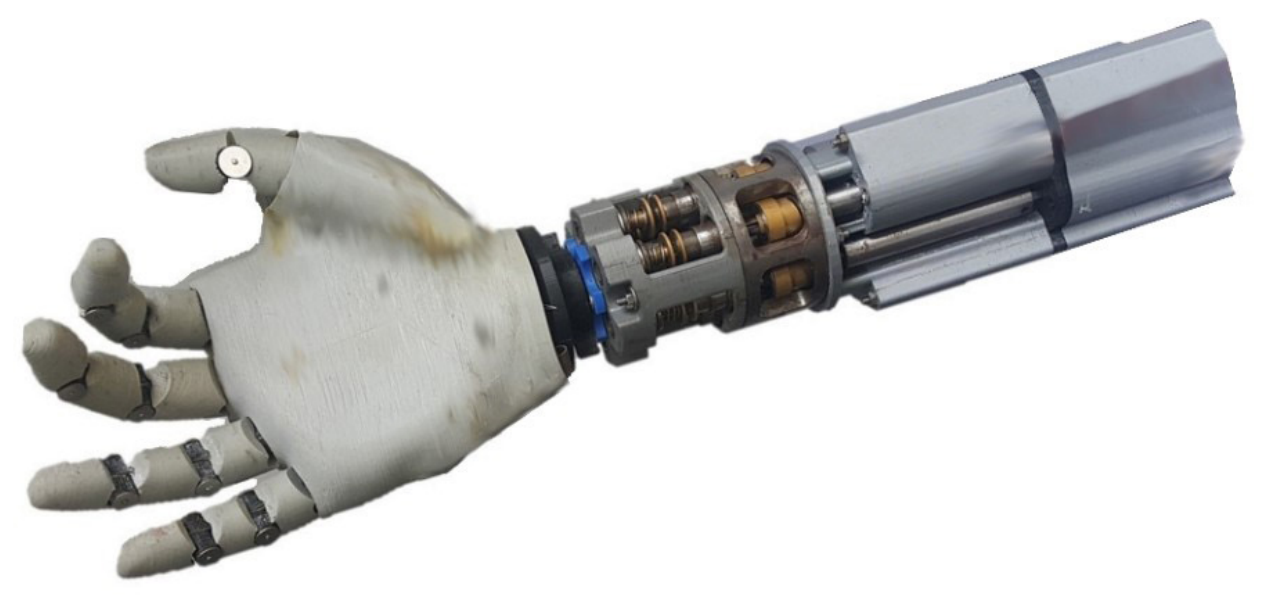

Figure 2. Prototype of the iGrasp Hand

To achieve the anatomical and functional aspects of a human hand, a prosthetic hand should imitate several salient features in addition to the flexion and extension of fingers. Recent research on transradial prostheses has focused on mimicking such features including but not limited to arching of the palm, function of the opposable thumb, and isometric hold of the hand. Yet, noticing a lack of research on devices with all three features above, the research team at the Bionics Laboratory came forward with the iGrasp Hand to bridge the gap.

One of the main features of the iGrasp Hand is its aesthetically attractive appearance resembling the human hand. The researchers have compacted the actuators and related mechanisms at the forearm section of the iGrasp Hand, facilitating the palm to acquire the geometry and the texture similar to the human hand. This has improved the dexterity of the iGrasp Hand.

The iGrasp Hand closely matches the anatomy of the human hand through its finger, thumb, and palm design. While the index and middle fingers of the iGrasp Hand possess three joints mimicking the human fingers, each additional joint at the ring and little fingers facilitates the arching of the palm. Three joints in the thumb of the iGrasp Hand can

\section{The motion analysis of the fingers of the iGrasp Hand revealed that the under-actuated} finger mechanism can adapt to the shape of the grasping object.<smiles>[124IH]</smiles>

provide flexion and extension motions similar to a biological hand, while an additional joint provides the functionality of the opposable thumbs.

The iGrasp Hand achieves motions similar to that of the human hand through a tendon-based operation. Five motors control the flexion-extension motions of the under-actuated fingers and the thumb 

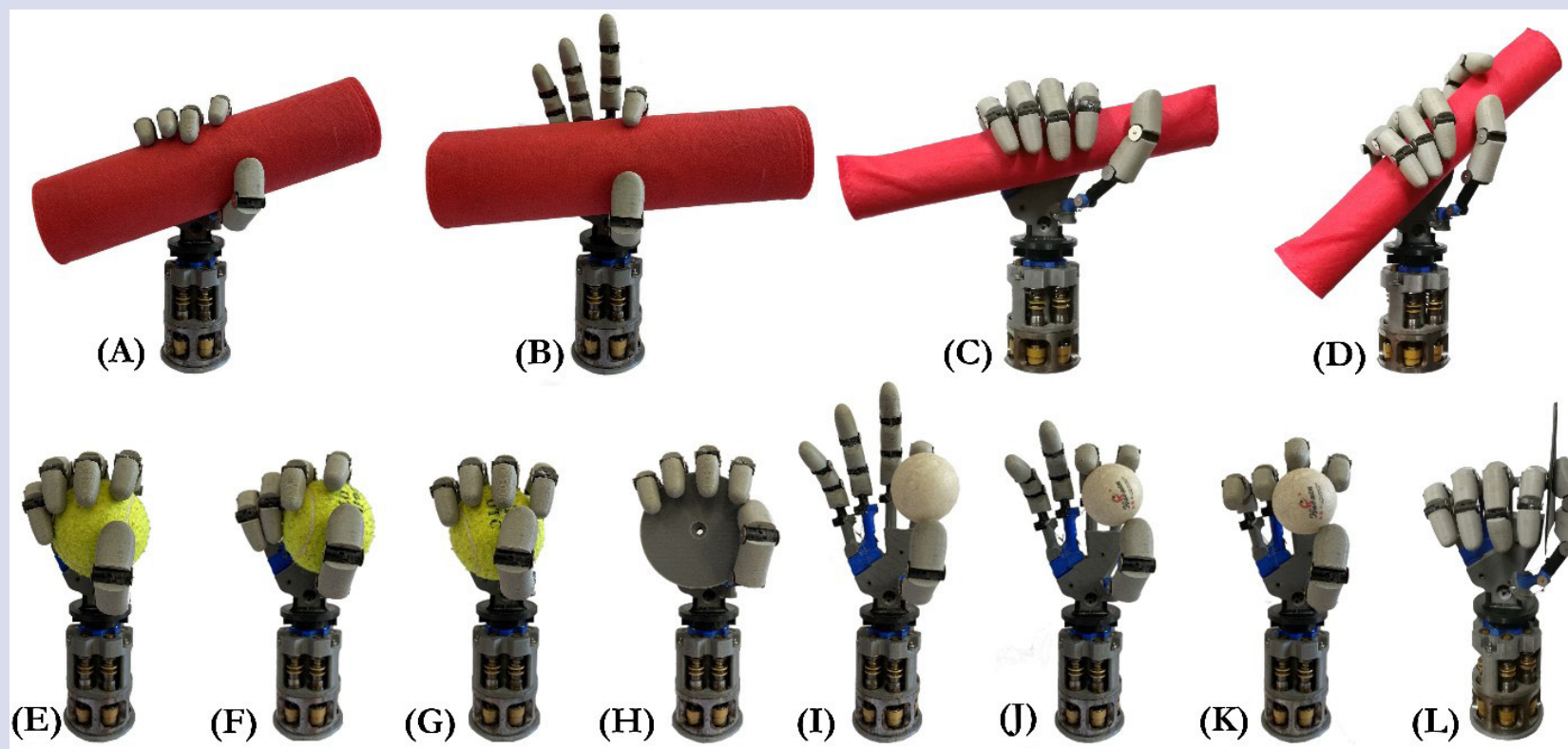

(H)
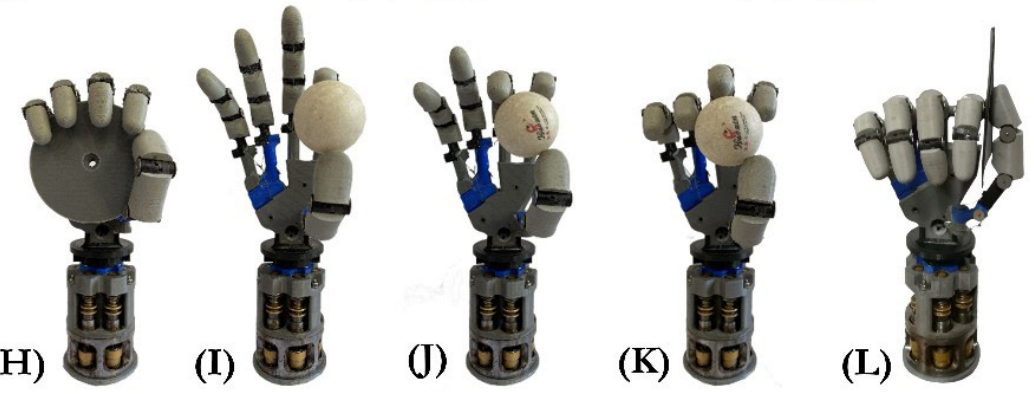

Figure 3. Grasping patterns achieved by iGrasp Hand; (A) Large diameter, (B) Ring, (K) Medium wrap, (L) Index finger extension, (C) Sphere 4-finger, (D) Sphere finger, (E) Power sphere, (F) Power disk, (G) Inferior pincer, (H) Tripod, (I) Quadpod, (K) Lateral pinch.

while a separate motor provides the motion for the opposable thumb function. Additionally, the torsional springs in the designed joints offer the passive stiffness required for adaptive grasping. The users can manually position the wrist joint orientation of the iGrasp Hand with their functional hand.

The research team has introduced a clutch unit for the iGrasp Hand to simulate the isometric holding of a human hand. The one-way-bearings incorporated in the clutch unit act as a locking mechanism that can sustain the grasp without the need of continuous actuation, thus reducing the power usage.

The fabrication of the prototype of the iGrasp Hand was an important step in evaluating the effectiveness of the designed prosthesis. The research team used the hand of a person matching the 50th percentile male population to obtain the anatomical structure of the hand and the palm geometry. They utilized these data to 3D print the structural components with Polylactic acid and the supple components with Thermoplastic Polyurethane while machining the other related components bringing the final mass of the prototype, excluding the power source, to $1.5 \mathrm{~kg}$.
The control system used, for the proof-of-concept of the iGrasp Hand, includes a model-based controller with closed-loop proportional-integralderivative (PID) control. An off-board desktop computer feeds the grasp labels in terms of motor rotation angles relevant to each grasping pattern to the microcontroller, which then drives the actuators through an intermediate motor controller. This control system facilitated the generation of motions and grasping patterns required for evaluating the performance of the iGrasp Hand.

During the performance evaluations, the iGrasp Hand demonstrated its ability to perform 12 power and precision grasps corresponding to the grasp taxonomy published in [4]. While the opposable thumb function of the iGrasp Hand supported inferior pincer, tripod, quadpod, and lateral pinch grasp patterns, the arching of the palm facilitated sphere four-finger, sphere finger, and index finger extension grasps. According to statistical analysis on the activities of daily living [5], the iGrasp Hand displayed the capability of performing more than $70 \%$ of the day to day tasks.

The research team evaluated the iGrasp Hand in terms of the Anthropomorphism Index of Mobility 
(AIM), which is an indicator that compares the mobility of a prosthesis with respect to that of the human hand. Considering factors such as the degrees of freedom and joints of the prosthetic hand, the ability to control these degrees of freedom independently, and the topology of the whole hand [6], the iGrasp Hand presented an AIM of $40 \%$. This is comparable with the commercially available robotic prostheses such as Michelangelo, Bebionic and iLimb whose AIMs are 14\%, 40\% and $45 \%$ respectively.

The motion analysis of the fingers of the iGrasp Hand revealed that the under-actuated finger mechanism can adapt to the shape of the grasping object. During the test conditions, the proximal digits of the fingers touched the cylindrical object first and then the distal digits came into contact by increasing the angle at the distal joint of the finger. This verified the ability of the iGrasp Hand to achieve adaptive grasping.

The research team further evaluated the power usage of the hand during the grasping and isometric hold positions. The results revealed that the current drawn by the actuators drops to zero at the isometric holding positions, thus verifying the power reduction capabilities of the iGrasp Hand.

The researchers at the Bionics laboratory of the Department of Mechanical Engineering have published a full account of the design and evaluation of the iGrasp Hand as a conference paper presented at the 2021 Moratuwa Engineering Research Conference [7].

iGrasp Hand is one research from many similar works done at the Bionics laboratory. Over the years, the undergraduate and graduate researchers affiliated to the Bionics laboratory have engaged in the development of many types of the upper limb and lower limb prostheses. Additionally, the laboratory has research of various calibre, focusing on the control of bionic devices through biological signals such as electromyography and electroencephalography. Several affiliated research teams have worked on exoskeletons targeting both rehabilitative and industrial applications.
As the research team of iGrasp Hand has proved yet again with their innovative transradial prosthesis design, the researchers from the Bionics laboratory continue research in the field of Bio-mechatronics to improve the lives of humans.

\section{References}

[1] "bebionic hand," [Online]. Available: https://www.ottobockus. com/prosthetics/upper-limb-prosthetics/solution-overview/bebionic-hand/,(accessed: Jul. 14, 2021).

[2] "ilimb hand," [Online]. Available:https://www.ossur.com/en-us/ pros-thetics/arms/i-limb-ultra, (accessed: Jul. 14, 2021).

[3] Michelangelo prosthetic hand," [Online]. Available:www.ottobockus.com/prosthetics/upper-limb-prosthetics/solution-overview/michelangelo-prosthetic-hand/, (accessed: Jul. 14, 2021).

[4] T. Feix, J. Romero, H. B. Schmiedmayer, A. M. Dollar, and D. Kragic, "The grasp taxonomy of human grasp types," IEEE Transactions on Human-Machine Systems, vol. 46, pp. 66-77, 2016.

[5] J.Z. Zheng, S. De La Rosa, and A. M. Dollar, "An investigation of grasp type and frequency in daily household and machine shop tasks," IEEE International Conference on Robotics and Automation, pp. 4169-4175, 2011.

[6] Llop-Harillo, A. P’erez-Gonz'alez, and V. Gracia-lb’ãnez, "Anthropomorphism index of mobility for artificial hands," Applied Bionics and Biomechanics, p. 1-11, 2019.

[7] N. P. Dasanayake, P.K.P. Viduranga, U.L.S. Perera, S.A.P.K. Siyambalagoda, T.M.G.C.S.P. Cooray, K.R.T. Fernando, R.K.P.S. Ranaweera and R.A.R.C. Gopura, "iGrasp Hand: A Biomimetic Transradial Robotic Hand Prosthesis with a Clutching Mechanism," Moratuwa Engineering Research Conference (MERCon), 2021

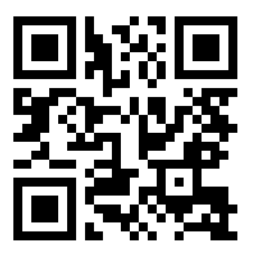

Article by

Kithmi Widanage, Shehara Perera, Nimantha Dasanayake, Prasad Viduranga, Pulitha Siyambalagoda, Sachin Cooray, Rushan Fernando, Pubudu Ranaweera and Ruwan Gopura

Bionics Laboratory, Department of Mechanical Engineering, Faculty of Engineering, University of Moratuwa, Sri Lanka 